\title{
Generation of extreme ultraviolet vector beams from infrared laser pulses
}

\author{
Alex Turpin ${ }^{1,2}$, Julio San Román ${ }^{3}$, Antonio Picón $^{3}$, Rokas Drevinskas $^{4}$, Ausra Cerkauskaite ${ }^{4}$ Peter G. \\ Kazansky ${ }^{4}$, Charles G. Durfee ${ }^{5}$, Íñigo J. Sola ${ }^{3}$ and Carlos Hernández-García ${ }^{3}$ \\ 1. Center of Advanced European Studies and Resarch (caesar), Ludwig-Erhard-Allee 2, 53175 Bonn, Germany \\ 2. Autonomous University of Barcelona, Edifici Cc Campus UAB, 08193 Bellaterra, Spain \\ 3. Grupo de Investigación en Aplicaciones del Láser y Fotónica, Física Aplicada, University of Salamanca, 37008 Salamanca, Spain \\ 4. Optoelectronics Research Centre, University of Southampton, UK \\ 5. Department of Physics, Colorado School of Mines, Golden, Colorado 80401, USA
}

Vector beams are light beams with spatially variant polarization [1]. During the last decade vector beams have become an indispensable tool in many areas of science and technology such as optical trapping, quantum memories, and quantum optics. In particular, radially and azimuthally polarized light beams are the paradigm of vector beams. Radial vector beams are especially interesting due to the non-vanishing longitudinal electric field component present in tightly focusing systems, which allows to sharply focus light below the diffraction limit [2]. On the other hand, azimuthal vector beams can induce longitudinal magnetic fields with potential applications in spectroscopy and microscopy. However, the spectral limitations of the generation techniques of vector beams based on linear optics prevent their efficient generation in the extreme-ultraviolet (EUV) and x-ray regimes, which would further extend their applications down to the nanometric scale. High-order harmonic generation (HHG) is known as a unique non-perturbative frequency up-conversion process for the generation of coherent EUV and soft x-ray radiation. A remarkable aspect of HHG is its fully coherent nature, mapping the characteristics of the driving field to the high frequency spectral region and thus allowing to harness the angular momentum properties of the harmonic radiation through modifications of the driving field [3-5].

In this work EUV vector beams are generated for the first time using HHG [6]. To do so, an infrared fs radially polarized vector beam -generated with a s-waveplate [7]- is focused into an argon gas target, whose atoms emit coherent EUV radiation through HHG. Our experimental and theoretical results demonstrate that HHG imprints the polarization state of the infrared beam, ranging from radial to azimuthal, into the higher frequency radiation. Our numerical simulations also demonstrate that the generated high-order harmonic beams can be synthesized into attosecond vector beams in the EUV/soft x-ray regime. Our proposal overcomes the state of the art limitations for the generation of vector beams far from the visible domain and could be applied in fields such as diffractive imaging, EUV lithography, or ultrafast control of magnetic properties.

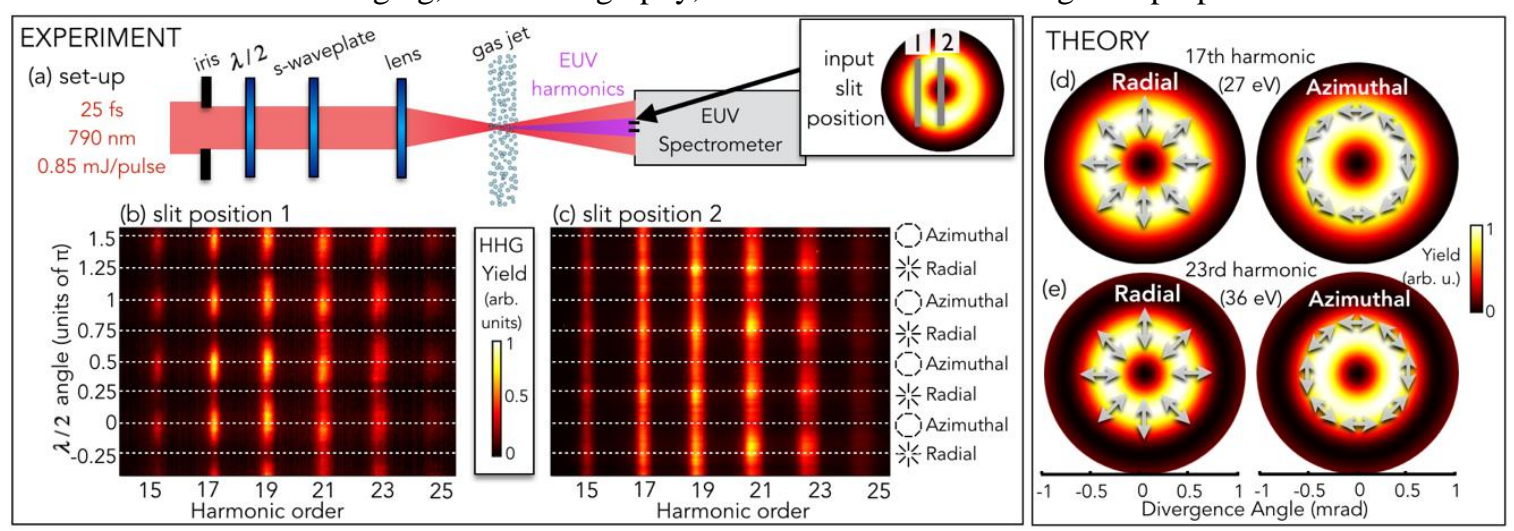

Fig. 1 Experimental set-up: an IR beam is converted into a vector beam after passing through a $\lambda / 2$ waveplate and the s-waveplate, and then focused into an Ar gas jet, where EUV harmonics are generated. The spectrometer input slit is placed at two different spatial positions of the harmonic beam, as indicated on the inset. The HHG spectra recorded at slit positions 1 (b) and 2 (b) are shown as a function of the $\lambda / 2$ angle. The $\pi / 2$ periodicity allows us to identify the cases where the harmonics are radially or azimuthally polarized. Theoretical intensity profiles for the (d) 17 th and (e) $23 \mathrm{rd}$ harmonics when driven by a radial (left) or azimuthal (right) IR beam.

\section{References}

[1] Q. Zhan, "Cylindrical vector beams: from mathematical concepts to applications," Adv. Opt. Photonics 1, 1-57 (2009).

[2] R. Dorn, S. Quabis, and G. Leuchs, “Sharper Focus for a Radially Polarized Light Beam,” Phys. Rev. Lett. 91, 233901 (2003).

[3] C. Hernández-García, A. Picón, J. San Román, and L. Plaja, "Attosecond Extreme Ultraviolet Vortices from High-Order Harmonic Generation,” Phys. Rev. Lett. 111, 083602 (2013).

[4] L. Rego, J. San Román, A. Picón, L. Plaja, and C. Hernández-García, "Nonperturbative Twist in the Generation of Extreme-Ultraviolet Vortex Beams", Phys. Rev. Lett. 117, 163202 (2016).

[5] A. Fleischer, O. Kfir, T. Diskin, P. Sidorenko, and O. Cohen, "Spin angular momentum and tunable polarization in high harmonic generation", Nat. Photonics 8 , 543-549 (2014).

[6] C. Hernández-García, A. Turpin, J. San Román, A. Picón, R. Drevinskas, A. Cerkauskaite, P. Kazansky, C. G. Durfee, and I. J. Sola, "Extreme Ultraviolet Vector Beams Driven by Infrared Laser Pulses", submitted.

[7] M. Beresna, M. Gecevicius, P. G. Kazansky, and T. Gertus, "Polarization sensitive elements fabricated by femtosecond laser nanostructuring of glass," Opt. Mater. Express 1, 783-795 (2011). 